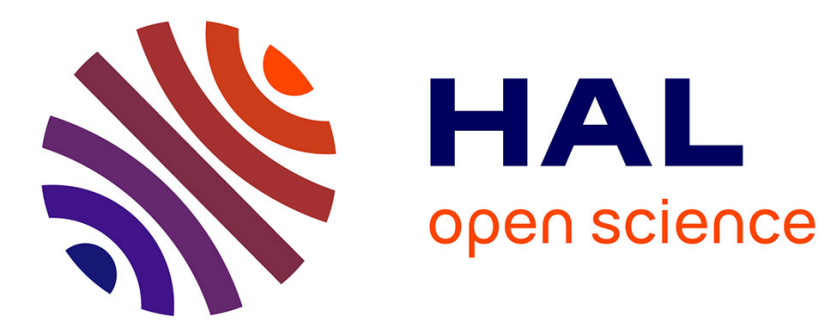

\title{
Conclusion - Bilan et perspectives
}

Valérie Bel, Frédérique Blaizot, Laurence Tranoy

\section{To cite this version:}

Valérie Bel, Frédérique Blaizot, Laurence Tranoy. Conclusion - Bilan et perspectives. Gallia Archéologie de la France antique, 2009, Pratiques et espaces funéraires de la Gaule durant l'Antiquité, 66 (1), pp.345-347. 10.3406/galia.2009.3378 . hal-01911025

\section{HAL Id: hal-01911025 \\ https://hal.science/hal-01911025}

Submitted on 7 Jan 2020

HAL is a multi-disciplinary open access archive for the deposit and dissemination of scientific research documents, whether they are published or not. The documents may come from teaching and research institutions in France or abroad, or from public or private research centers.
L'archive ouverte pluridisciplinaire HAL, est destinée au dépôt et à la diffusion de documents scientifiques de niveau recherche, publiés ou non, émanant des établissements d'enseignement et de recherche français ou étrangers, des laboratoires publics ou privés.

\section{(ㅇ)(1) $\$$}

Distributed under a Creative Commons Attribution - NonCommercial - NoDerivatives 44.0 


\title{
Conclusion
}

\section{BILAN ET PERSPECTIVES}

\author{
Valérie BEL, Frédérique BLAIZOT et Laurence TRANOY
}

Nous avons tenté ici de clarifier les configurations archéologiques rencontrées pour proposer une restitution du déroulement le plus probable des rites funéraires. Les récentes données de l'archéologie préventive nous ont amenées à nous interroger sur le bien-fondé de nos démarches ; avions-nous sélectionné les bons critères ? Le regard que nous portions sur les données, ou plus exactement le mode de classement de ces dernières, était-il valide ? Enfin, les références techno-culturelles qui sont les nôtres, et de fait, nos schémas interprétatifs sont-ils adaptés aux réalités de la société antique ? Dans un premier temps, la mise en commun de nos informations a au moins contribué à mettre en évidence la complexité des situations. En effet, l'interprétation des structures ainsi que la valeur et le rôle accordés au mobilier sont les expressions majeures d'une démarche traditionnellement fondée sur des a priori et des raisonnements circulaires ; la confrontation des données permet aujourd'hui d'invalider ces schémas. Ainsi il nous a fallu rompre avec l'idée qu'à une structure qui nous apparaît spécifique correspond une interprétation unique (bûcher, tombe, dépôt, tombe-bûcher ) et avec celle qu'un espace funéraire est forcément constitué d'un ensemble de structures qui fonctionnent individuellement. Parmi elles, celles qui s'avèrent être ni des tombes ni des bûchers, souvent marginalisés lors des fouilles et des études, ont été réintégrées dans le circuit des gestes liés aux cérémonies des enterrements et des commémorations (fosses dites dépotoirs par exemple). En examinant l'organisation interne des espaces que les communautés ont accordé à leurs morts, il est apparu que la compréhension de ces lieux était indissociable d'une analyse plus globale du contexte spatial dans lequel ils s'inscrivent et évoluent, qu'il s'agisse du monde rural ou du suburbium. Les données analysées ont fait long feu de la notion de "nécropole », au sens littéral du terme, pour nos régions, et mettent plutôt en lumière une occupation funéraire, par petits ensembles, sur un mode saltatoire. En réinterprétant les marqueurs classiques, il a été possible de faire émerger un système qui reste largement perfectible et sujet à discussions.

Au terme de cette synthèse, qui, comme toujours, tire les grandes lignes d'une pléthore de données, nous restons très conscientes de la difficulté à dégager un modèle, puisque nous avons presque autant de configurations que de sites. En l'état actuel des connaissances, nous manquons de données homogènes à grande échelle pour évaluer les différences de comportement entre les différents secteurs géographiques (notamment entre les civitates) et les interpréter.

Il ressort des données exploitées dans ce dossier que beaucoup de sites a priori bien conservés et donc avec un potentiel scientifique important ne peuvent pas être traités avec les moyens dont on dispose. D’un autre côté, une grande part de sites fouillés et étudiés possèdent un potentiel informatif réduit du fait d'une mauvaise conservation ou de la petite taille de la surface d'investigation. Cruel dilemme. Faut-il encourager le retour à une politique moins interventionniste qui privilégierait les sites d'exception ? La question n'est pas simple car, de cette manière, on se prive d'une approche spatiale de l'occupation qui, 
en dépit de sites de qualité inégale, apporte assurément une connaissance fondamentale sur l'organisation des sociétés. Les ensembles incomplets ou mal conservés sont en outre susceptibles d'offrir, sur des points particuliers du rituel, des éléments d'information ponctuels mais décisifs pour la compréhension des pratiques funéraires.

Il nous apparaît néanmoins de plus en plus nécessaire de pouvoir nous consacrer à quelques études menées exhaustivement sur d'importantes séries ciblées qui renferment un potentiel à l'aulne de nos questionnements. À l'heure actuelle, les coûts engendrés par l'étude d'une grande série, qui demande à mettre en œuvre un lourd protocole d'analyse, rendent cet objectif inaccessible. Il appert que les très grands ensembles funéraires fouillés ne peuvent presque quasi jamais être exploités, ce qui réduit les possibilités de tester nos hypothèses et d'ouvrir encore le champ de nos questions.

Dans plusieurs régions, des sites d'envergure restent à publier. En Lorraine, on peut évoquer celui de Salmon à Cutry en Meurthe-et-Moselle (800 dépôts de crémation des $\mathrm{I}^{\mathrm{er}}$ - III ${ }^{\mathrm{e}}$ s. et près de 300 inhumations tardo-antiques et altomédiévales), de Qui Qu'en Grogne à Toul dans le même département (140 ha concernant des complexes agraires, des établissements, des espaces funéraires et des sépultures s'échelonnant de l'âge du Fer jusqu'au III ${ }^{\mathrm{e}}$ s.), d'Hottwiese et Bitche à Epping en Moselle (environ $6000 \mathrm{~m}^{2}$ d'occupation funéraire du ${ }^{\mathrm{er}} \mathrm{s}$. av. J.-C. au $\mathrm{III}^{\mathrm{e}} \mathrm{s}$. de notre ère) ou celui de la Croix-Guillaume à Saint-Quirin, également en Moselle, constitué de plusieurs noyaux funéraires des $\mathrm{I}^{\mathrm{er}}-\mathrm{III}{ }^{\mathrm{e}} \mathrm{s}$. En Champagne-Ardenne, les sites du Puisard à Caurel, de Thibault des Murs à Châlons-en-Champagne et des Monts à Plichancourt dans la Marne, constituent des séries majeures pour l'étude des pratiques funéraires, tandis que l'Alsace recèle un potentiel important sur la commune de Strasbourg (notamment dans le quartier de Koenigshoffen : site des Capucins à Hohberg), malheureusement fouillé anciennement de manière inégale. En Franche-Comté, une nouvelle analyse des rites funéraires serait à mener sur la base du corpus étudié par L. Joan dans le cadre de sa thèse, tandis que le site d'Au Velours, à Poligny (Jura), représenté par un ensemble funéraire mis en place sur un sanctuaire au $\mathrm{I}^{\mathrm{er}} \mathrm{s}$. qui se développe jusqu'au $\mathrm{IV}^{\mathrm{e}} \mathrm{s}$. à partir d'une tombe fondatrice, reste à étudier. En Bourgogne, des sites exceptionnels comme ceux du parking SainteAnne à Dijon, de Derrière la Cure à Vertault, des Grands Bois de Nod à Nod-sur-Seine (Côte-d'Or) et du parking Rambuteau à Mâcon (Saône-et-Loire), nécessiteraient plus que les quelques présentations générales qui en ont été effectuées pour l'instant. Prochainement, le rapport de fouilles de Pont-l'Évêque, à Saint-Pantaléon, devrait fournir les premières données cohérentes sur les ensembles funéraires périurbains d'Autun (Saône-et-Loire). En Picardie, la publication exhaustive des Sables à Limé dans l'Aisne (245 inhumations et 90 structures liées à la crémation datées de La Tène finale au IV ${ }^{\mathrm{e}}$ s. apr. J.-C.) (Soupart, 2001) et, dans le Nord - Pas-de-Calais, celle des Oblets à Thérouanne (Bura, 2001) sont très attendues, tout comme la publication exhaustive, en Rhône-Alpes, de la Favorite à Lyon (Tranoy, 1995b) et, dans l'Ouest, celles des ensembles funéraires de Saintes et du mausolée de Naintré (Vienne). Des données récentes seront disponibles aussi à Poitiers (Vienne), avec la fouille du quartier de l'hypogée des Dunes. En MidiPyrénées, on mentionnera par exemple les découvertes toulousaines ou encore l'ensemble funéraire de Cayrac et celui de la Vayssière à L'Hospitalet-du-Larzac, dans l'Aveyron (230 structures liées à la pratique de la crémation). La région Provence-Alpes-Côte d'Azur a également livré plusieurs grandes séries périurbaines dont les ensembles du Pauvadou et de Saint-Lambert à Fréjus (Var) ou celle de la Rouguière à Riez (Alpes-de-Haute-Provence).

Le nombre de sites en souffrance ne témoigne pas seulement de la difficulté à exploiter ce type d'ensembles. Il résulte aussi bien souvent d'une inadéquation entre les stratégies et les méthodes mises en œuvre et le potentiel réel du site et ses caractéristiques propres. Par exemple, certaines structures complexes ou de grandes dimensions (vastes aires de crémation ou zones de rejet) restent encore mal comprises car elles n'ont pas fait l'objet de fouilles adaptées. En la matière, une fouille exhaustive mal conduite peut se révéler moins utile qu'une approche par échantillonnage selon un protocole adéquat rigoureux et orienté en opérant des choix destinés à répondre à des questions précises.

Il arrive bien souvent que les études soient menées sans tenir compte des caractéristiques du site. Ainsi, des études très précises sont-elles engagées sur des lots d'ossements issus de dépôts incomplets. On peut déplorer que fréquemment la vaisselle soit analysée dans une perspective purement typo-chronologique, sans aucune considération sur sa fonction dans les pratiques funéraires. Des études spécialisées parfois très lourdes sont engagées sans réflexion commune orientée vers la compréhension des vestiges. Elles s'ajoutent les unes aux autres et leurs résultats sont parfois même publiés hors de leur contexte.

Selon nous, la fouille des ensembles sépulcraux doit être en priorité centrée sur les problématiques funéraires puisque, on l'a vu, la caractérisation du système funéraire 
conditionne l'interprétation sociale et culturelle des sépultures. L'opération archéologique doit ainsi s'attacher à définir la place accordée aux morts (où sont-ils ? quelles sont les règles qui régissent l'utilisation des espaces funéraires ?), à caractériser le mode de traitement du défunt, à identifier les différentes étapes du rituel et les gestes associés à chacune d'entre elles. Si, dans ce domaine, chaque détail compte, il est impératif de mettre en place une démarche globale et de fonder la réflexion sur l'ensemble des éléments présents dans l'espace funéraire. Ainsi seront largement améliorées notre connaissance des rites élaborés autour de la mort et, par là même, notre approche des sociétés antiques. 